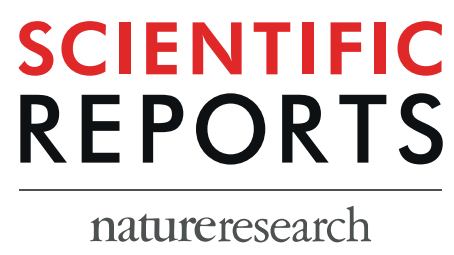

\title{
OPEN Myc/Max dependent intronic long antisense noncoding RNA, EVA1A-AS, suppresses the expression of Myc/Max dependent anti-proliferating gene EVA1A in a U2 dependent manner
}

\author{
Svenja E. Niehus ${ }^{1,4}$, Aldrige B. Allister ${ }^{1,4}$, Andrea Hoffmann ${ }^{2}$, Lutz Wiehlmann ${ }^{3}$, \\ Teruko Tamura ${ }^{1} \&$ Doan Duy Hai Tran ${ }^{1 *}$
}

The Myc gene has been implicated in the pathogenesis of most types of human cancerous tumors. Myc/Max activates large numbers of pro-tumor genes; however it also induces anti-proliferation genes. When anti-proliferation genes are activated by Myc, cancer cells can only survive if they are downregulated. Hepatocellular carcinoma (HCC) specific intronic long noncoding antisense (Inc-AS) RNA, the EVA1A-AS gene, is located within the second intron (12) of the EVA1A gene (EVA-1 homolog A) that encodes an anti-proliferation factor. Indeed, EVA1A, but not EVA1A-AS, is expressed in normal liver. Depletion of EVA1A-AS suppressed cell proliferation of HepG2 cells by upregulation of EVA1A. Overexpression of EVA1A caused cell death at the G2/M phase via microtubule catastrophe. Furthermore, suppressed EVA1A expression levels are negatively correlated with differentiation grade in 365 primary HCCs, while EVA1A-AS expression levels are positively correlated with patient survival. Notably, both EVA1A and EVA1A-AS were activated by the Myc/Max complex. Eva1A-AS is transcribed in the opposite direction near the 3 'splice site of EVA1A 12. The second intron did not splice out in a U2 dependent manner and EVA1A mRNA is not exported. Thus, the Myc/Max dependent anti-proliferating gene, EVA1A, is controlled by Myc/Max dependent anti-sense noncoding RNA for HCC survival.

Noncoding RNAs regulate a broad spectrum of cellular processes, including oncogenic signaling. Recent deep sequencing studies determined that the number of long noncoding RNAs is 2- to 3-fold greater than protein coding RNAs ${ }^{1}$. Furthermore, by analyzing RNA-seq data from 20 HCC patients, 8603 long noncoding RNA (lncRNAs) were recently identified ${ }^{2}$. Notably, approximately $76 \%$ of these were unannotated ${ }^{2}$, suggesting that the expression of IncRNAs is highly cell type and cancer type specific.

Comprehensive expression analysis of the Encyclopedia of DNA Elements Consortium (ENCODE) indicated that three-quarters of the human genome is capable of being transcribed, but only about $1.5 \%$ of this fraction contains the genome code for proteins ${ }^{3,4}$

We have recently shown that upon depletion of THOC5, a member of the TREX (transcription/export) complex 396 genes were commonly downregulated in HCC cell lines, Huh7 and HepG2, and those affected cells then underwent apoptosis ${ }^{5}$. Here, depletion of Linc00176, one of the THOC5 target long noncoding RNAs, resulted in cell death in $\mathrm{HCC}^{6}$. We then proceeded to search for THOC5 target long noncoding RNAs for HCC maintenance.

${ }^{1}$ Institut fuer Biochemie, OE4310, Medizinische Hochschule Hannover, Carl-Neuberg-Str. 1, D-30623, Hannover, Germany. ${ }^{2}$ Klinik für Orthopädie OE8893, Medizinische Hochschule Hannover, Stadtfelddamm 34, D-30625, Hannover, Germany. ${ }^{3}$ Zentrale Forschungseinrichtung Genomics OE 9415, Medizinische Hochschule Hannover, CarlNeuberg-Str. 1, D-30623, Hannover, Germany. ${ }^{4}$ These authors contributed equally: Svenja E. Niehus and Aldrige B. Allister. *email:Tran.Doan@MH-Hannover.de 
In this study we have characterized one of these THOC5 target antisense (AS) long noncoding genes, EVA1A (eva-1homolog A)/TMEM166 (transmembrane protein 166)-antisense (EVA1A-AS). Both EVA1A and EVA1A-AS genes are activated by the Myc/Max complex that is the most commonly overexpressed gene in human cancers. We show that the intronic AS lncRNA, EVA1A-AS, suppressed EVA1A expression and EVA1A is highly expressed in normal liver, but not in HCCs. Overexpression of EVA1A as well as depletion of EVA1A-AS induced inhibiting cell proliferation and cell death in HCC. Here EVA1A-AS suppressed splicing of EVA1A intron 2 in a U2 dependent manner. These data imply that EVA1A-AS participates in the survival of HCC that expresses EVA1A.

\section{Results}

EVA1A-AS is expressed in HepG2 cells, but not in normal hepatocytes and suppresses EVA1A expression. We have previously shown that depletion of THOC5, an mRNA export complex protein, in HCC downregulates only a subset of genes, but causes cell death ${ }^{5}$. One of the essential THOC5 target genes for HCC maintenance is a long noncoding RNA, Linc $00176^{6}$. These data imply that additional THOC5 target long noncoding RNAs may participate in HCC development and survival. To identify further THOC5 target long noncoding RNAs, HepG2 cells were infected with lentivirus carrying shTHOC5 or shCr (control) for 3 days. Under these conditions, THOC5 protein was downregulated to approximately $50 \%$ (Fig. 1A) and previously identified THOC5 target coding genes, inhibitor of DNA binding 1 (ID1) and NDC80 kinetochore complex component (SPC25), were also downregulated more than 2-fold (Fig. 1A). RNAs were then applied for RNA sequencing. The sequencing data of THOC5 revealed that all exon reads were clearly suppressed in THOC5 depleted cells (Fig. 1B).

The 3208 annotated long noncoding RNAs were downregulated more than 2-fold upon depletion of THOC5. These long noncoding RNA transcripts were further filtered for the following criteria: 1) expression of more than 100 FPKM (Fragments Per Kilobase of Exon Per Million Fragments Mapped) in shCr virus infected HepG2, 2) predicted length of RNA more than 500 nucleotides (nt), 3) expression of less than 50 FPKM in other tissues (Human BodyMap 2.0 data from Illumina). The 6 lncRNAs, namely NONHSAG028257.2, NONHSAG094271.1, NONHSAG030119.2, NONHSAG075906.1, NONHSAG015030.2, and NONHSAG035576.2, were selected using these criteria. We then further characterized the 2 long antisense ncRNAs (NONHSAG028257.2, and NONHSAG094271.1). NONHSAG028257.2 is an antisense noncoding RNA to the EVA1A gene, whereas NONHSAG094271.1 is an antisense noncoding RNA to the KRT19P1 pseudogene with unknown function.

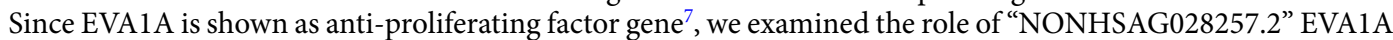
antisense (AS) noncoding RNA in HCC (Fig. 1C). EVA1A-AS is located in the second intron (I2) of the EVA-1A gene (Fig. 1C). Notably, our sequencing data reveals that depletion of THOC5 reduced the expression level of EVA1A-AS, whereas in contrast EVA1A expression is enhanced (Fig. 1C), suggesting that these two genes may regulate each other. Indeed, depletion of EVA1A or EVA1A-AS by treatment with specific siRNAs in HepG2 cells enhanced the expression level of EVA1A-AS or EVA1A, respectively (Fig. 1D-F). EVA1A protein is also upregulated in EVA1A-AS depleted cells and the protein was expressed at low level in siCr treated HepG2 cells (Fig. 1G), indicating that EVA1A-AS is a suppressor for EVA1A expression.

Depletion of EVA1A-AS in HepG2 cells induced lipid droplet accumulation and stopped the cell cycle, but additional depletion of EVA1A rescued cell proliferation. Upon depletion of EVA1A-AS, large vacuoles and lipid droplets were observed within 3 days (Fig. 2A). Lipid droplets were not detected within the vacuoles. Importantly, these cells become significantly larger than cells transfected with siCr, and these large cells simultaneously lose the expression of proliferation marker Ki67 (Fig. 2A,B). Upon additional depletion of EVA1A, siEVA1A-AS transfected cells significantly regained Ki67 expression (Fig. 2A,B, p=3.0E-07). Here, upon depletion of EVA1A alone, the phenotype of HepG2 was not significantly altered (Fig. 2A). To examine the subcellular localization of endogenous EVA1A, we stained HepG2 cells with anti EVA1A antibody before and after depletion of EVA1A-AS. Endogenous EVA1A is located mainly at the perinuclear region in untreated cells, but upon depletion of EVA1A-AS, EVA1A is distributed throughout the cytoplasm in punctate forms that are similar to lipid droplets by ORO staining (Fig. 2A,C), and EVA1A is partially co-localized with lipid droplets.

To examine whether EVA1A and EVA1A-AS control cell growth, we examined cell growth based on crystal violet staining. In agreement with Ki67 staining data, upon depletion with EVA1A-AS, not EVA1A, cell growth was approximately $50 \%$ reduced within 3 days (Fig. 2D). Cell growth of siEVA1A-AS transfected cells was recovered when cells were further transfected with siEVA1A (Fig. 2D), while overexpression of EVA1A suppressed cell growth (Fig. 2E).

To examine which cell cycle phase is influenced by overexpression of EVA1A, propidium iodide (PI) labeled cells were analyzed by FACS (Fig. 2F). G2 peak of EVA1A overexpressing HepG2 cells was drastically reduced (Fig. 2F), suggesting that EVA1A caused G1-S arrest or cell death in G2-M phase. We then examined cell cycle of EVA1A overexpressing cells and control HepG2 cells in the presence or absence of the blocker for G2/M phase CDK-1 kinase inhibitor, RO3306 $(5 \mu \mathrm{M})$. Both cells went G2 phase (Fig. 2F), suggesting that EVA1A overexpressing cells did not go to G1-S arrest.

Ectopic expression of EVA1A in HCC cells caused cell death after G2 phase. To examine whether overexpression of EVA1A causes cell death in HepG2 cells, we next expressed GFP-EVA1A in HepG2 cells. Half of the GFP-EVA1A positive cells became TUNEL positive within $24 \mathrm{~h}$ after transfection, while expression of GFP alone resulted in less than 5\% TUNEL positive cells (Fig. 3A,B). We obtained similar data using Myc-tagged EVA1A (SI Fig. 1A). Apoptosis markers, such as cleavage of PARP or caspase 3 were not detected in EVA1A overexpressing HepG2 cells, and MLKL was not phosphorylated in these cells (SI Fig. 2). 


\section{A}
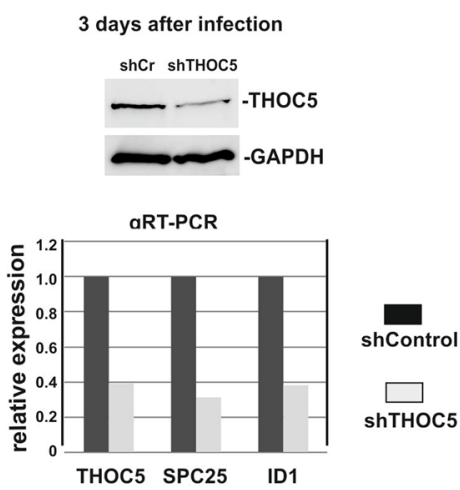

C

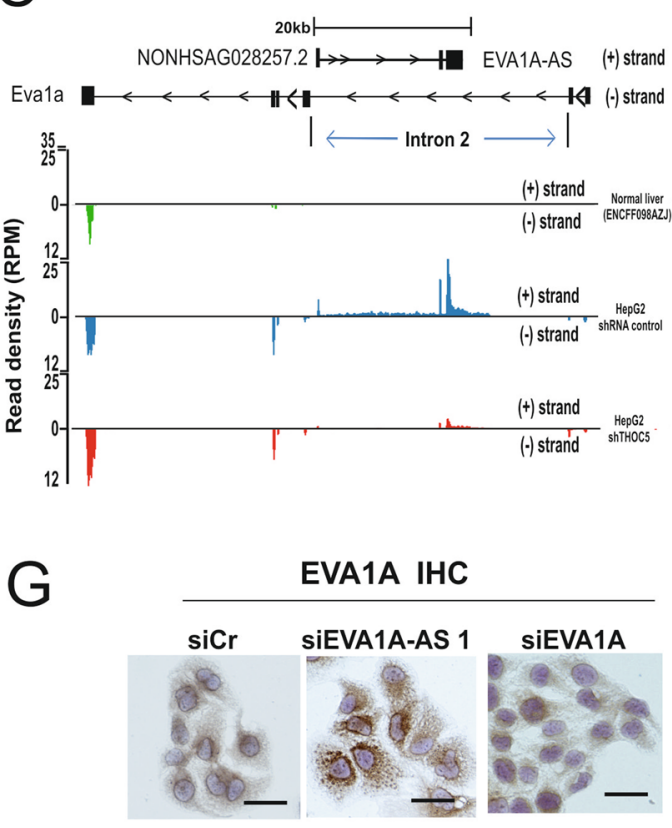

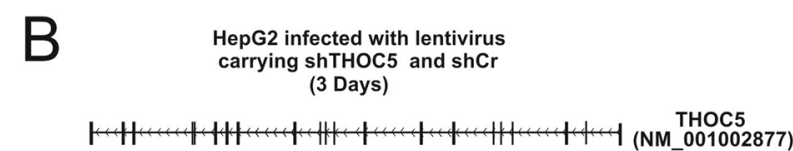
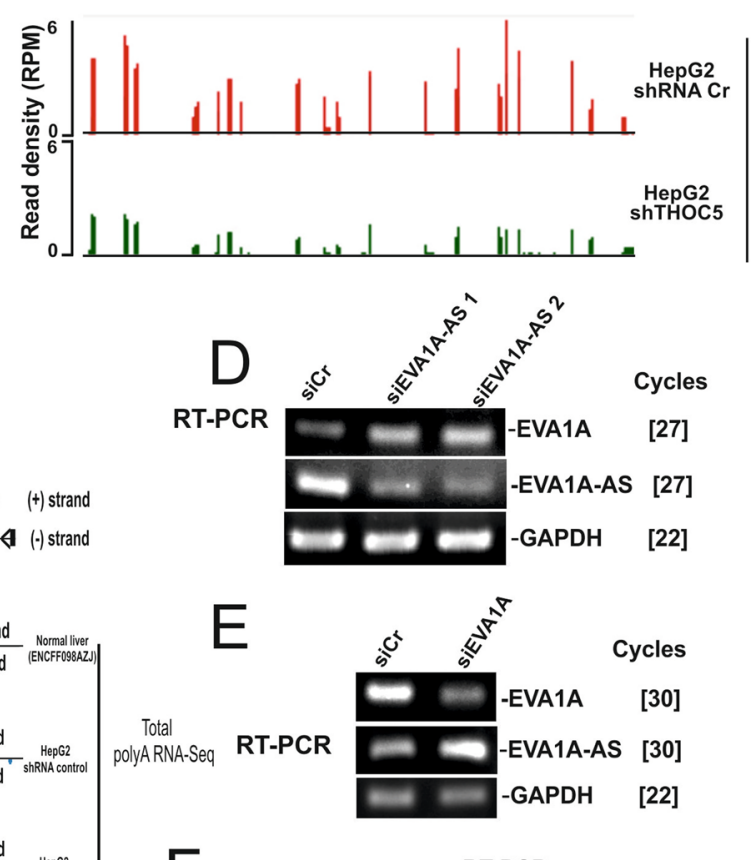

$\mathrm{F}$

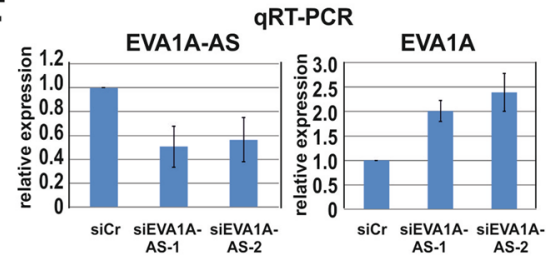

I

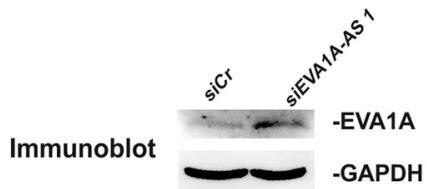

Figure 1. EVA1A-AS is expressed in HepG2 cells, but not in normal hepatocytes and suppresses EVA1A expression. HepG2 cells were infected with lentivirus carrying shTHOC5 (shTHOC5) or sh control (shCr) for three days. (A) Cell lysates were applied for THOC5 specific immunoblot. GAPDH specific immunoblot was performed as loading control. RNAs were isolated from a sister culture and applied for THOC5, SPC25, ID1 specific qRT-PCR. (B) RNA sequence data of THOC5. SeqMonk was used to quantitate and visualize the data. Read density is shown as reads per million (RPM). (C) Predicted EVA1A-AS (NONHSAG028257.2) (+strand) and EVA1A (-strand) in HepG2 cells infected with lentivirus carrying shRNA control or shTHOC5. Total RNA-seq datasets from human liver tissue (ENCFF098AZJ) were generated by the ENCODE Consortium and were aligned to the reference human genome (GRCh38). Black box: exon. (D) RNAs were isolated from HepG2 treated with siCr, siEVA1A-AS-1, or siEVA1A-AS-2 and were supplied for EVA1A, EVA1A-AS or GAPDH specific semi-quantitative RT-PCR. []: number of PCR cycles. (E) RNAs were isolated from HepG2 cells treated with siCr, or siEVA1A and supplied for EVA1A-AS, EVA1A or GAPDH specific semi-quantitative RT-PCR. []: number of PCR cycles. (F) RNAs were isolated from sister culture of (D) and applied for EVA1A-AS, EVA1A specific q-RT-PCR. Three independent experiments were performed. Bars represent $+/-\mathrm{SD}$. (G) Cell extracts from a sister culture of $(\mathbf{D})$ were applied for EVA1A and GAPDH specific immunoblot.

To examine whether EVA1A exerted an influence on the transition of G2/M phase, EVA1A overexpressing cells were treated with CDK-1 kinase inhibitor, RO3306 $(5 \mu \mathrm{M})$ for $24 \mathrm{~h}$, whereafter the inhibitor was removed. This treatment did not result in a change to the endogenous expression level of EVA1A, whereas RO3306 treatment suppressed EVA1A-AS expression. After removal of the inhibitor, however, the expression level of EVA1A-AS recovered within 90 min (Fig. 3C). Although RO3306 treated EVA1A positive cells increased in size, no TUNEL positive cells were observed (Fig. 3D), whereas removal of RO3306 induced TUNEL positive cells within 10 to $30 \mathrm{~min}$ (Fig. 3D). In addition, typical spindles were not observed by tubulin specific staining after the removal of the inhibitor in EVA1A overexpressing HepG2 cells (Fig. 3E), indicating that cells died during 
A
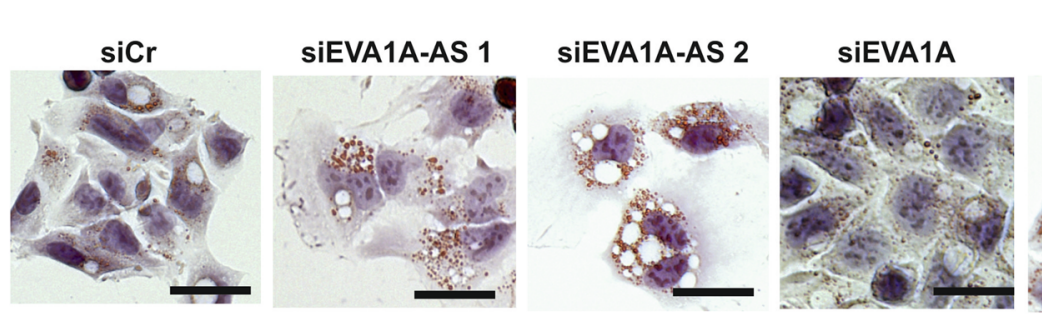

SIEVA1A-AS 1 SIEVA1A

ORO
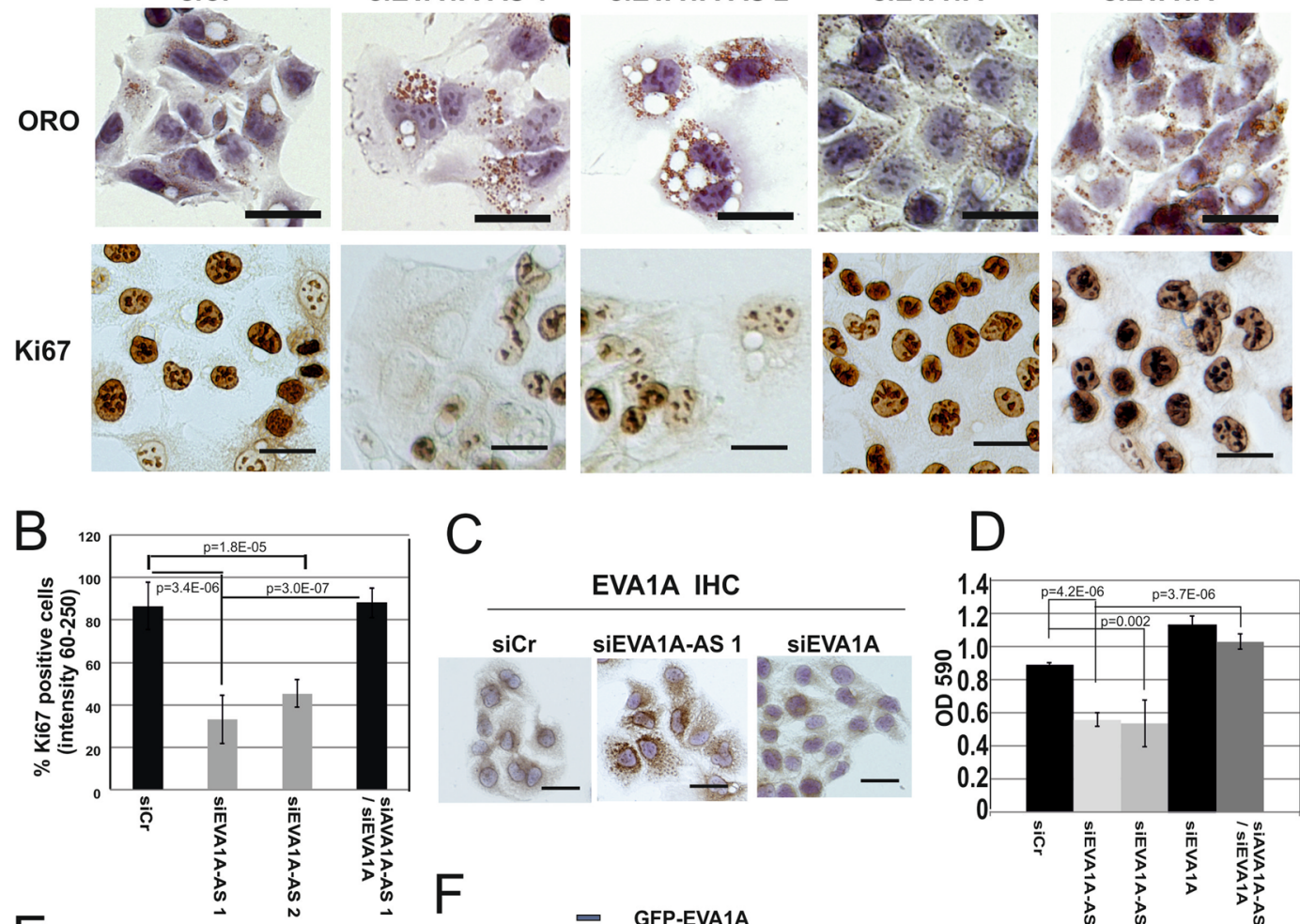

C
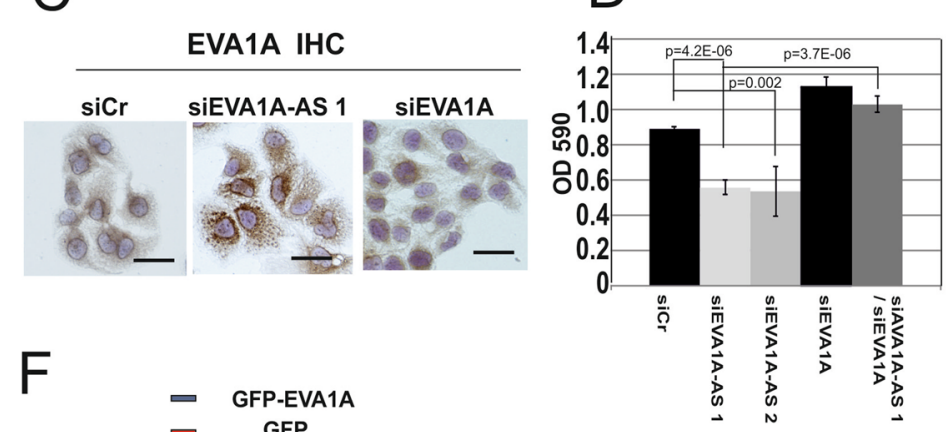

$E$
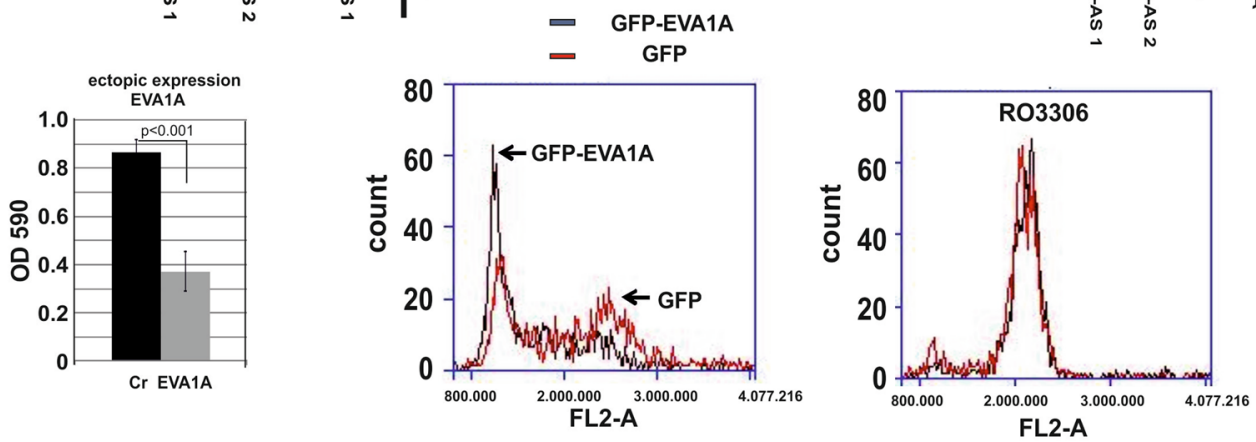

Figure 2. Depletion of EVA1A-AS in HepG2 cells induced lipid droplet accumulation and stopped cell cycle. (A) HepG2 cells were chemically fixed 3 days after transfection with siCr, siEVA1A-AS 1, siEVA1A-AS 2 and/or siEVA1A and then applied for oil red O (ORO) or Ki67 specific immunohistochemical staining. Bars represent $40 \mu \mathrm{m}$. Three independent experiments were performed and representative images are shown. (B) To quantitate the intensity of Ki67 staining, reciprocal pixel intensity was determined by subtracting the Ki67 intensity from the maximum pixel intensity in white unstained areas (as measured by the mean intensity function in the Nikon NIS elements D 3.0 Software). The mean percentage of dark staining (positive: reciprocal intensity 60-250) nuclei is shown. Bars represent $+/-$ SD. At least 200 cells from 3 images were quantitated per experiment. $p$ value: t-test. (C) Endogenous EVA1A was immunohistochemically stained in HepG2 cells after transfection with siCr, siEVA1A-AS, or siEVA1A. Bars represent $40 \mu \mathrm{m}$. (D) Sister culture of (A) was stained with crystal violet and measured density by OD590. (E) EVA1A was ectopically expressed in HepG2 cells and three days after transfection cells were fixed and stained with crystal violet. (F) HepG2 cells were transfected with GFP or GFP-EVA1A in the presence or absence of CDK1 inhibitor, RO3306. Fixed cells were stained with propidium iodide (PI) and analysed by Accuri-C6 flow cytometer (BD Biosciences) under standard settings for detecting PI (FL-2) using a $488 \mathrm{~nm}$ laser.

G2/M transition by microtubule catastrophe ${ }^{8}$. Notably, treatment of HepG2 cells with CDK1 inhibitor caused lipid droplet accumulation (Fig. 3F).

EVA1A expression level is correlated with the differentiation grade of HCC. We next examined whether EVA1A and EVA1A-AS are expressed in normal liver and four HCC cell lines, HepG2, Huh7, Hep3B and HLE. EVA1A was expressed in all samples, however the expression level of EVA1A in HepG2 and Huh7 is approximately 5-fold less than in normal hepatocytes, while EVA1A-AS is expressed in HepG2, HepB3 and HLE cells (differentiation grade 2-3 (G2-G3) but not in Huh7 cells (differentiation grade 1 (G1)) or normal hepatocytes 

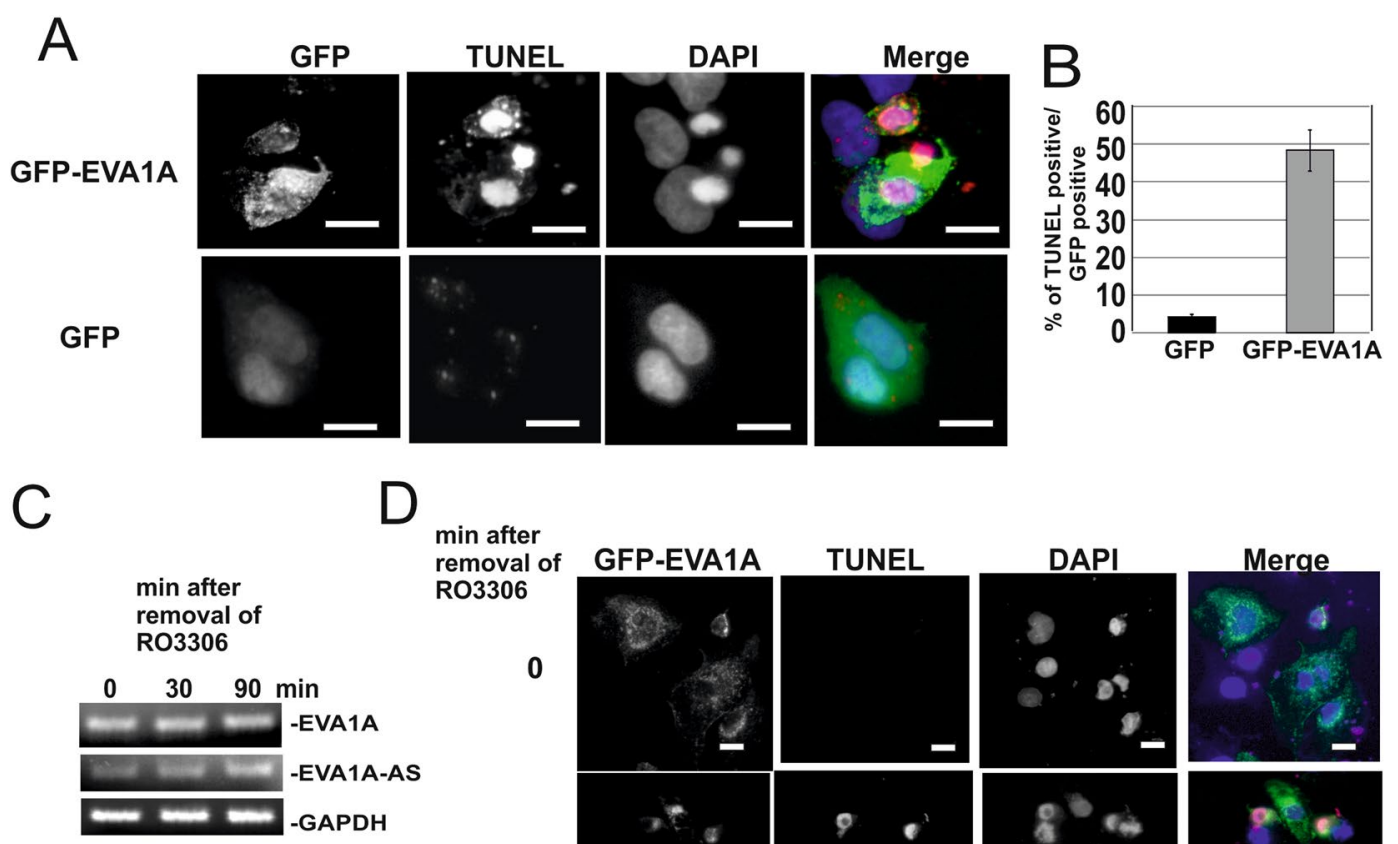

min after RO3306
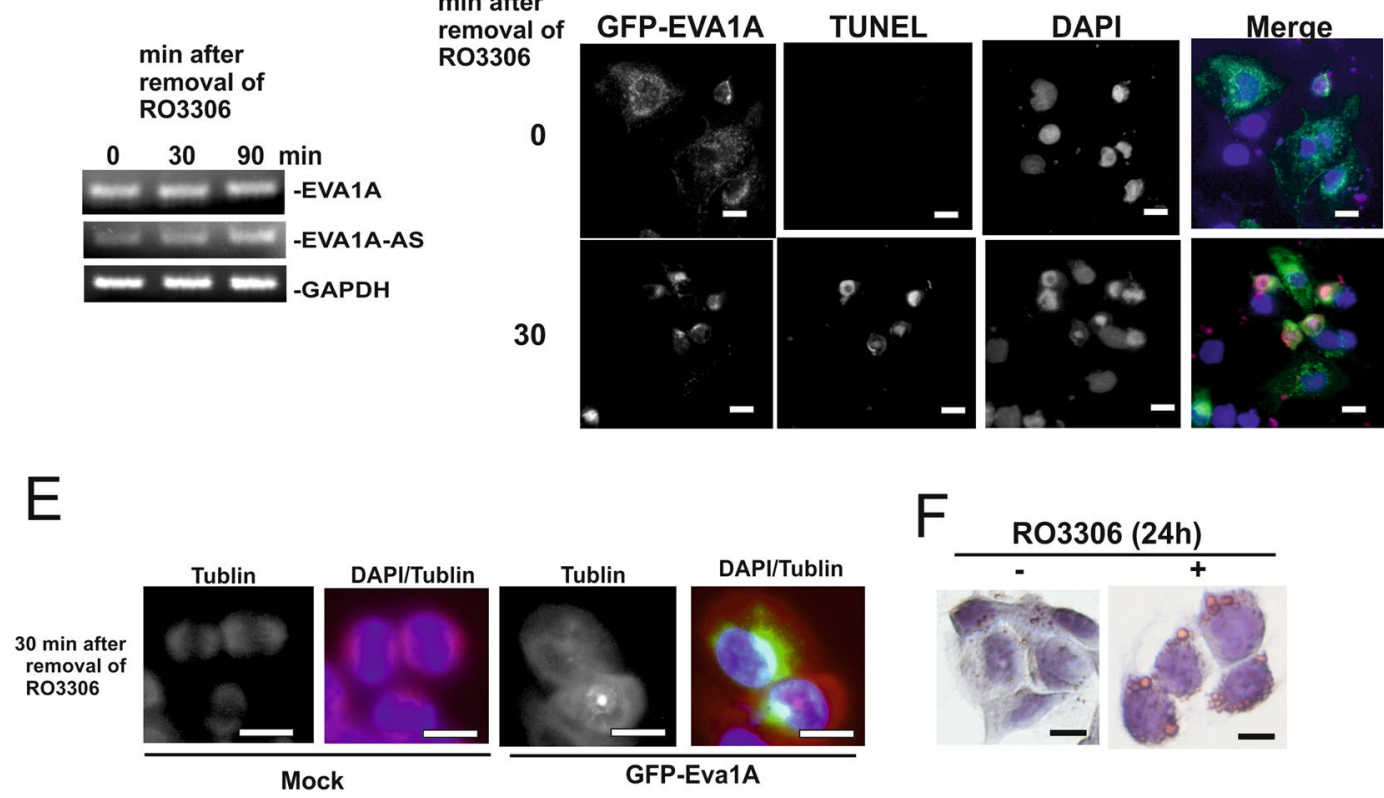

Figure 3. Ectopic expression of EVA1A in HCC cells caused cell death. (AB) GFP-EVA1A or GFP was overexpressed in HepG2 cells and applied for TUNEL (red), and DAPI (blue) staining. Bars represent $40 \mu \mathrm{m}$. (A) The ratio between GFP: TUNEL positive cells was determined. (B) (C) HepG2 cells were treated with CDK1 kinase inhibitor RO3306 $(5 \mu \mathrm{M})$ for $24 \mathrm{~h}$. Total RNAs obtained from cells after removal of inhibitor $(0,30$, and $120 \mathrm{~min}$ ) were applied for EVA1A, EVA1A-AS or GAPDH specific semi quantitative RT-PCR as indicated. (D,E) HepG2 cells were transfected with GFP-EVA1A cDNA and then treated with CDK1 kinase inhibitor RO3306 $(5 \mu \mathrm{M})$ for $24 \mathrm{~h}$. Cells were fixed 0 or $30 \mathrm{~min}$ after removal of inhibitor. Cells were stained by DAPI and TUNEL (D) or Tubulin (E) antibody and DAPI. Three independent experiments were performed and representative images are shown. Bars represent $40 \mu \mathrm{m}$. (F) HepG2 cells were incubated with (+) or without (-) RO3306 for $24 \mathrm{~h}$. Cells were stained by ORO. Bars represent $40 \mu \mathrm{m}$.

(Fig. 4A). In primary HCC EVA1A-AS expression was observed in only a subset of G2 and G3 HCCs (Fig. 4B), while EVA1A gene expression was significantly suppressed in $G 2$ to $G 4$ primary HCCs $(p=0.0009-p=3.2 E-12)$ (Fig. 4C). These data suggest that EVA1 A may act as a negative regulator of HCC survival. Indeed, the EVA1A-AS expression level is also significantly correlated with HCC patient survival time. We analyzed the data by means of the Kaplan-Meier estimation. Correlation between EVA1A-AS expression levels (FPKM $>0(n=41): F P K M=0$ $(\mathrm{n}=16)$ ) and survival time (within 1 year) is significant (Fig. $4 \mathrm{D}$, log rank test: $\mathrm{p}=0.0799)$. These data suggest that EVA1A-AS participates in growth and/or survival of HCC in cell lines as well as in primary HCCs.

We next examined whether a point mutation of p53 correlates with EVA1A-AS expression. In HCC cell lines, HepG2 has p53 without a mutation and in Huh7 and HLE cells p53 is point mutated. EVA1A-AS is expressed in HepG2, and HLE, but not in Huh7 (Fig. 4A), indicating that EVA1A-AS expression is not correlated with a p53 mutation. In agreement with these data, in primary HCC $40-50 \%$ of p53 are mutated in both HCC with or without EVA1A-AS expression (Data obtained from the cancer genome atlas).

Both EVA1A-AS and EVA1A are regulated by the Myc/Max complex. We next examined the manner in which EVA1A-AS is transcriptionally regulated. DNase-sequencing (ENCFF591XCX) and cap analysis of gene expression (CAGE) (ENCFF177HHM) data suggest that the promoter of EVA1A-AS is located on "GRCh38 Chr2: 75516378-75524065” in HepG2 (Fig. 5A, yellow box). ENCODE ChIP-seq data revealed that Myc 

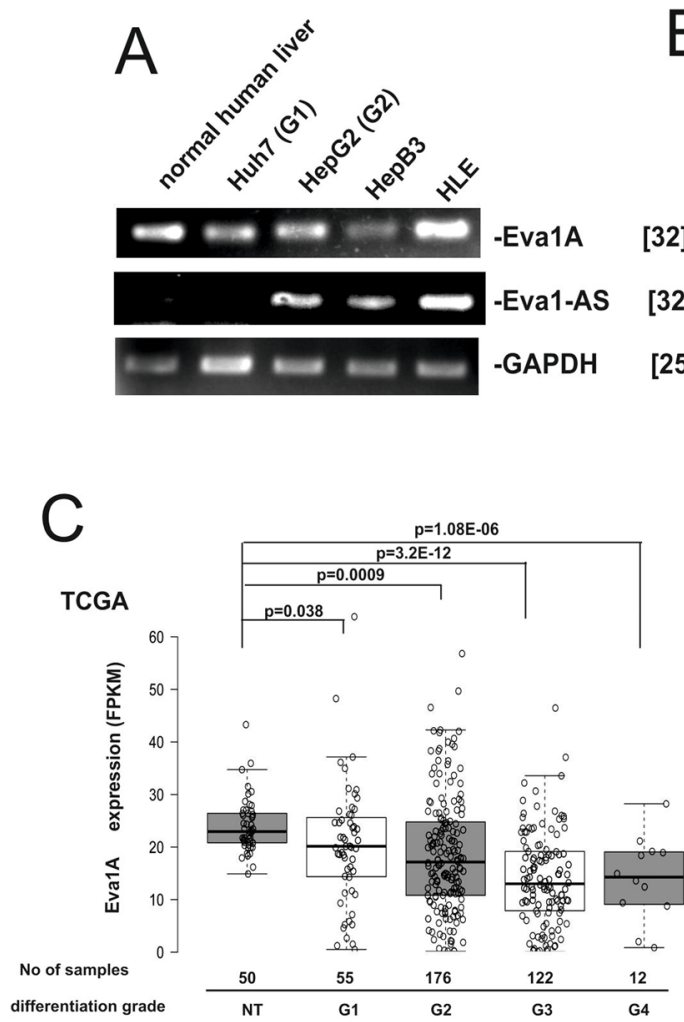

[32]

[25]

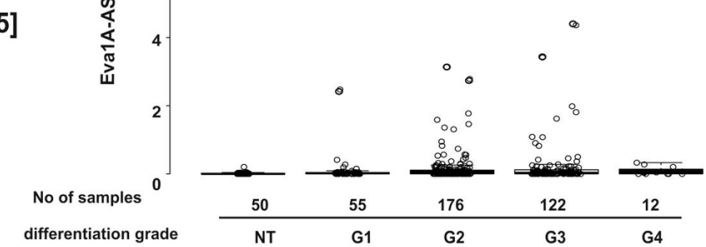

Overall Survival Kaplan-Meier Estimation

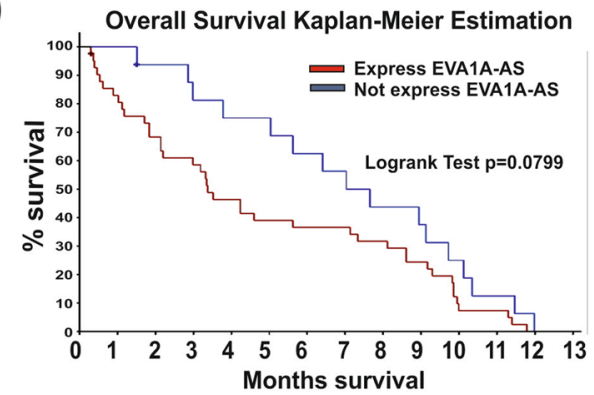

Figure 4. The EVA1A expression level is correlated with the differentiation grade of HCC. (A) Total RNAs from Huh7 (G1), HepG2 (G2), HepB3 (G3) or HLE cells were supplied for EVA1A, EVA1A-AS and GAPDH specific semi-quantitative RT-PCR. Normal human liver RNA was purchased from human normal hepatocyte (Origene, Maryland, USA). []: number of PCR cycles. Three independent experiments were performed. (B-D) Expression of EVA1A-AS (B) and EVA1A (C) in 365 primary HCCs (differentiation grade 1-4) and 50 normal liver samples. Data obtained from the cancer genome atlas (TCGA) (https://cancergenome.nih.gov/) shown as box blot. p value: t-test between G1, G2, G3 and G4 HCCs and 50 corresponding normal livers. (D) Correlation between EVA1A-AS expression level $(F P K M>0(n=41): F P K M=0(n=16))$ and survival time (within 12 months) is shown using Kaplan-Meier estimation (Log rank test: $\mathrm{p}=0.0799)$.

(ENCFF263DIF) and Max (ENCFF000PKZ) bind to this position and Mxil (ENCFF000XUX), a negative regulator for $\mathrm{Myc} / \mathrm{Max}^{9}$, binds to a much lesser extent (Fig. 5A). In agreement with these data, upon depletion of Max and/or Myc, the EVA1A-AS expression level is downregulated more than 4-fold (Fig. 5B,C), however depletion of Mxi1, suppressor of Max/Myc, upregulated EVA1A-AS expression (Fig. 5C). Strikingly, Myc- and Max-CHIP-seq data suggest that the EVA1A promoter is also regulated by Myc (Fig. 5A, green box). Upon knockdown of Myc and/or Max, EVA1A expression is downregulated. Depletion of Mxil results in upregulation of EVA1A (Fig. 5C).

To confirm these data, we next performed a Myc- and Max- specific chromatin immunoprecipitation (CHIP) assay. In HepG2 cells, Myc and Max bound to both proximal promoter regions of Eva1A and Eva1A-AS, while in Huh7 cells Myc and Max bound to EVA1A, but not to the EVA1A-AS promoter region (Fig. 5D). EVA1A-AS and EVA1A proximal promoter regions contain $9 \mathrm{x}$ and $1 \mathrm{x}$ E-BOX (CANNTG), respectively (Fig. 5E). To determine whether these putative promoters are indeed activated by Myc, both proximal promoter regions were fused to the luciferase gene and the reporter assay was performed in the presence or absence of $\mathrm{Myc}^{10}$. In the presence of Myc, EVA1A-AS and EVA1A putative promoters were activated 14- and 3.3 -fold in the absence of Myc, respectively (Fig. 5E).

These data imply that Myc/Max induces both the EVA1A and EVA1A-AS genes and since EVA1A is an anti-survival protein in HCC, EVA1A-AS suppressed the expression of EVA1A, resulting in the survival of HCC.

EVA1A-AS suppressed EVA1A expression by inhibiting the splicing of intron 2 (I2) of EVA1A. EVA1A-AS is transcribed in the opposite direction near the $3^{\prime}$ splice site of EVA1A I2 (Fig. 6A), suggesting that it may suppress the splicing of EVA1A I2. To examine whether EVA1A-AS exerts an influence on the EVA1A splicing event, we applied primer pairs located in Exon (E) 1, I2, E3 and E6 for RT-PCR or qRT-PCR in the siCr, siEVA1A-AS-1 or siEVA1-AS-2 treated HepG2 cells (Fig. 6B,C). When a primer pair that was located in E1-E3 (primers F1-B2) was used, we observed predominantly a $210 \mathrm{nt}$ long PCR product instead of $298 \mathrm{nt}$ in all samples. The $210 \mathrm{nt}$ bands contain only E1 and E3 (Fig. 6C), implying that E2 is spliced out independently from EVA1A-AS in HepG2 cells. An intron containing EVA1A (using a primer pair located in I2 and E3) accumulated $>3$-fold in the nuclear fraction in siCr cells than in EVA1A-AS depleted cells (Fig. 6C). EVA1A mRNA is exported $>2$-fold in EVA1A-AS depleted cells than in siCr cells (Fig. 6D). These data suggest that EVA1A-AS 

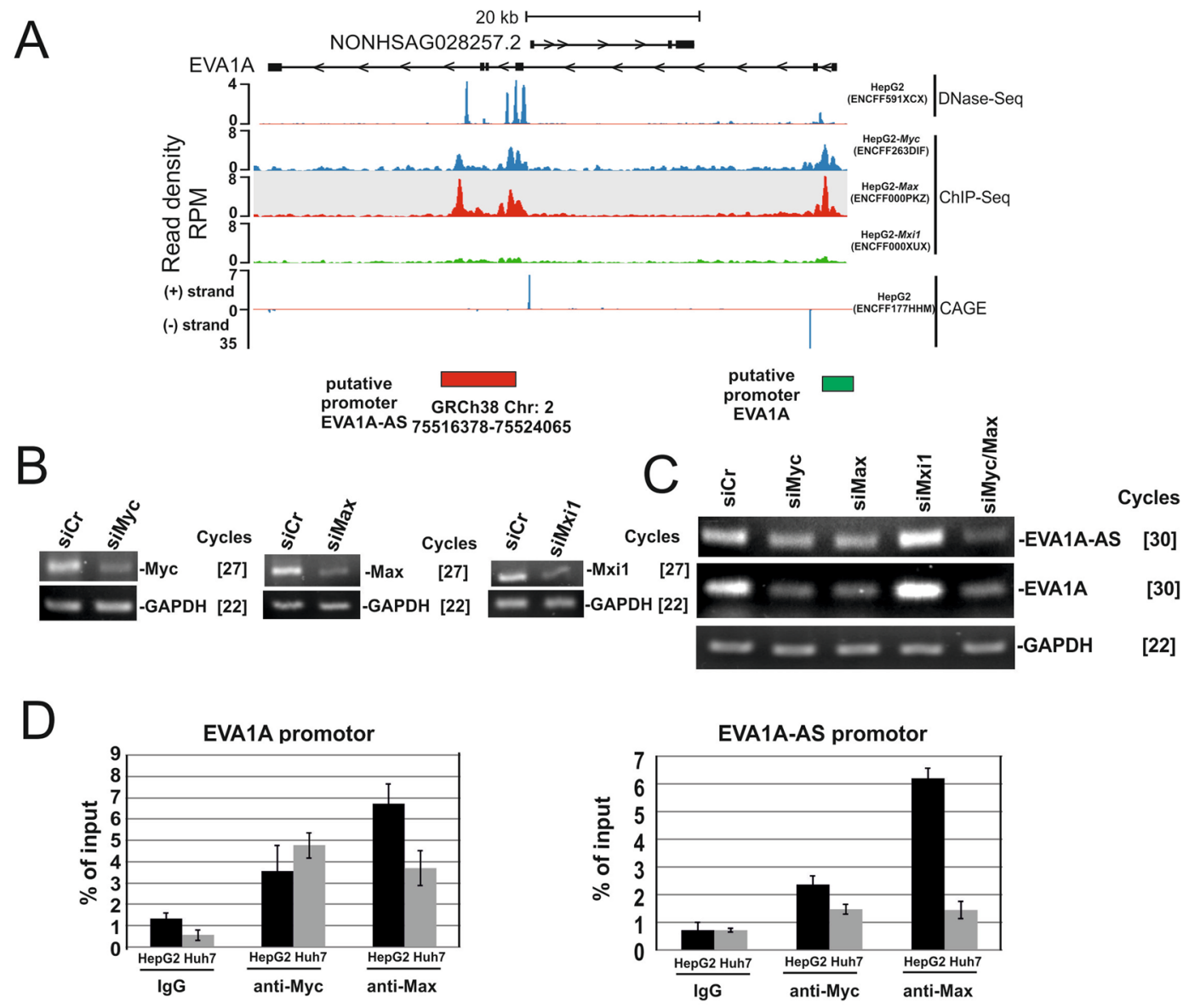

E putative promoter

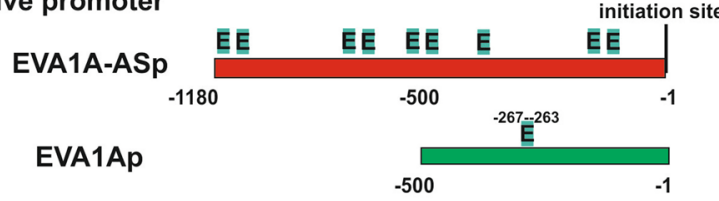

E: E-Box

CANNTG

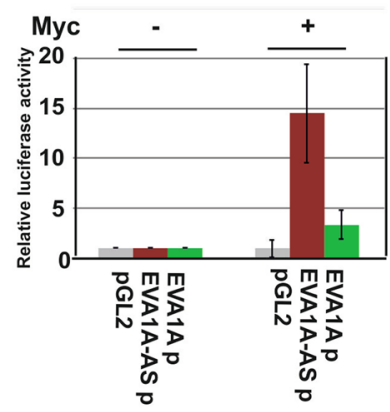

Figure 5. Both EVA1A and EVA1A-AS were regulated by Myc/Max. (A) DNase-sequencing (DNase-seq) datasets from HepG2 (ENCFF591XCX), ChIP-seq datasets HepG2 Myc (ENCFF263DIF), HepG2 Max (ENCFF000PKZ), and HepG2 Mxi1 (ENCFF000XUX), and cap analysis of gene expression (CAGE) data in HepG2 cells (ENCFF177HHM) generated by the ENCODE Consortium were aligned to the reference human genome (GRCh38). SeqMonk was used to quantitate and visualize the data. Yellow box: putative EVA1A-AS promoter; green box: putative EVA1A promoter. (B,C) siCr, siMyc, siMax, or siMxil, were transfected in HepG2 cells and RNA was applied for Myc, Max, Mxi1, EVA1A-AS, EVA1A or GAPDH specific semi quantitative qRTPCR as indicated. []: number of PCR cycles. Three independent experiments were performed and representative images are shown. (D) After cross-linking by adding formaldehyde, protein and DNA were extracted from HepG2 or Huh7 cells and the chromatin was sheared by sonication. Cell extracts and immunoprecipitates using Myc or Max antibodies or control IgG were analyzed by EVA1A (promoter region, -124 to -299) and EVA1A-AS (promoter region, -42 to -235 ) specific PCR (Table 1; ChIP). Numbers represent nucleotide numbers from the initiation site for each gene. Data represent percent (\%) input of each PCR reaction. Three independent experiments were performed. (E) location of E-Box (E: CANNTG) in the putative promoter regions of EVA1A-AS (EVA1A-ASp) and EVA1A (EVA1Ap). Putative promoter region of EVA1A-AS and EVA1A fused luciferase and luciferase activity was examined in the presence or absence of Myc.

impaired splicing of I2. We next analyzed the EVA1A-AS sequence to determine whether it could potentially form an RNA duplex with the U2 binding site. Notably, EVA1A-AS nascent RNA contains 4x UCAUAU (Fig. 6B) which are complementary to the branch point site sequence (AUAUGAU), predicted by branching point prediction 


\begin{tabular}{|c|c|c|c|c|c|c|c|}
\hline Gene & Accession number & Forward primer & Reverse primer & $\begin{array}{l}\text { RT- } \\
\text { PCR }\end{array}$ & $\begin{array}{l}\text { qRT- } \\
\text { PCR }\end{array}$ & ChIP & \begin{tabular}{|l} 
PCR \\
product \\
(bp)
\end{tabular} \\
\hline EVA1A & NM_001135032.1 & ATGCCCCTTTTCTAGCCAGG & CAGTCGCGTTTCTCCGATCT & $\mathrm{x}$ & $\mathrm{x}$ & & 153 \\
\hline EVA1A-AS & NR_110281.1 & CCTGCATCACTGCATTTCCG & TGCGAAAGAGTGGCACACAG & $\mathrm{x}$ & $\mathrm{x}$ & & 145 \\
\hline GAPDH & NM_002046 & TGTTGCCATCAATGACCCCTT & CTCCACGACGTACTCAGCG & $\mathrm{x}$ & $\mathrm{x}$ & & 202 \\
\hline ID1 & NM_002165.3 & CTCTGCACACCTACTAGTCACC & GTCACGTTTGGTGCTTCTCAGATTTC & & $\mathrm{x}$ & & 117 \\
\hline Max & NM_002382 & CAATCTGCGGCTGACAAACG & GCACTTGACCTCGCCTTCT & $\mathrm{x}$ & & & 272 \\
\hline Mxil & NM_130439 & GCGCCTTTGTTTAGAACGCTT & AATGCTGTCCATTCGTATTCGT & $\mathrm{x}$ & & & 235 \\
\hline$M y c$ & NM_002467.4 & CATGAGGAGACACCGCCCACCACCAG & TGCGTAGTTGTGCTGATGTGTGGAGACGTG & $\mathrm{x}$ & & & 210 \\
\hline$S P C 25$ & NM_020675 & TCATCTTGAGGGCCTAGCAG & CATTGTGCACATGTACCCTAAAAC & & $\mathrm{x}$ & & 221 \\
\hline THOC5 & NM_001002878 & GAGACCCTCACCAGCAAACAC & CGAATGGCATAAACAGGTACTCC & & $\mathrm{x}$ & & 213 \\
\hline$F 1-B 2$ & & TTTGTCGTGTCCGCCCCTCAG & TGTCAGGAGCAGAGAAGTTTCTG & $\mathrm{x}$ & & & 210 \\
\hline$F 2-B 1$ & & CTGGACATAGTGGCATATAACTGTAG & GATGATGAACTCAGTTTCAGATATGTTG & $\mathrm{x}$ & & & 242 \\
\hline$F 3-B 2$ & & GTATGACCCTGGGAGACTGG & TGTCAGGAGCAGAGAAGTTTCTG & $\mathrm{x}$ & $\mathrm{x}$ & & 329 \\
\hline F4-B3 & & ATGCCCCTTTTCTAGCCAGG & CAGTCGCGTTTCTCCGATCT & $\mathrm{x}$ & $\mathrm{x}$ & & 153 \\
\hline EVA1A promoter & & GCCGTTCAGGTTTCGCGTCTT & CAAGCCCCGCAGAAGCTGCA & & & $\mathrm{x}$ & 176 \\
\hline EVA1A-AS promoter & & СТСАСТCTCCAATCGGAACCTCTA & CTCATGAGAACTCACCCACTATTAG & & & $\mathrm{x}$ & 193 \\
\hline
\end{tabular}

Table 1. PCR primer pair sequences for selected genes. EVA1A: eva-1 homolog A, regulator of programmed cell death; EVA1A-AS: eva-1 antisense RNA; GAPDH: glyceraldehyde-3 phosphate dehydrogenase; ID1: inhibitor of DNA binding 1, HLH protein; Max: MYC associated factor X; Mxi1: MAX-interacting protein 1; Myc: v-myc avian myelocytomatosis viral oncogene homolog; SPC25: NDC80 kinetochore complex component; THOC5: THO complex subunit 5 homolog.

$(\mathrm{BPP})^{11}$ recognized by U2 snRNA in the I2 of EVA1A (Fig. 6B). To determine whether EVA1A and EVA1A-AS form a double-stranded RNA, we treated RNA from HepG2 cell with shortcut RNase III and then analyzed the undigested RNA. Upon treatment with RNaseIII, the amount of I2-E3 (intron containing transcript) was approximately $20 \%$ of that from the untreated RNA, while the amount of the E6 product was not changed (Fig. 6E), suggesting that I2 but not E6 RNA forms a double stranded RNA. Furthermore, treatment with the U2 inhibitor, U2-AMO (Gene Tools LLC, Oregon, USA), reduced the effect on I2 splicing by depletion of EVA1A-AS (Fig. 6F).

To further examine whether EVA1A-AS suppresses the recruitment of the U2 complex to the $3^{\prime}$ splicing site of I2, we performed an RNA immunoprecipitation (RIP) assay using an antibody against the splicing factor 3a subunit 1 (SF3A1), a component of the mature U2 small nuclear ribonucleoprotein particle (snRNP), in siCr or siEVA1A-AS transfected cells. SF3A1 is expressed in similar amounts in both transfected cells (Fig. 6G), however using a primer pair located in 2 and E3 we showed that SF3A1 binds significantly stronger $(\mathrm{p}=0.02)$ to EVA1A pre-mRNA in the absence of EVA1A-AS (Fig. 6G), suggesting that EVA1A-AS alters splicing of EVA1A by reducing the recruitment of $\mathrm{U} 2$ snRNP to EVA1A pre-mRNA.

\section{Discussion}

The Myc gene has been implicated in the pathogenesis of most types of human cancerous tumors ${ }^{12}$. Notably, Myc induced not only pro oncogenic genes but also anti oncogenic genes. We have recently shown that Myc/ Max dependent tumor suppressor miRNAs, miR9-5p and miR185-5p were inactivated via long noncoding RNA:Linc00176-sponge ${ }^{6}$. Linc00176 is also a Myc dependent gene ${ }^{6}$. In addition, some Myc inducible long noncoding RNAs participate in suppression of anti-oncogenic gene expression. We further examined additional Myc dependent antisense lncRNAs and their potential target coding RNAs that are downregulated in HCCs. Notably, in HepG2 cells at least 5 Myc dependent antisense noncoding RNAs are detected within Myc dependent coding genes, such as Flavin-containing monooxygenase (FMO) $5^{13}$, zinc finger protein (ZFP) $69^{14}$, erythroblast membrane-associated protein (ERMAP) 26, and inhibitor of DNA binding (ID) $2^{15}$. In addition, expression of these coding genes is suppressed in HCC, suggesting that these genes may negatively participate in cancer formation.

How does antisense RNA suppress splicing of sense RNA? It has been recently shown that several natural antisense transcripts (NATs) such as SAF and Zeb2-NAT lncRNAs altered the recruitment of members of the spliceosome to sense transcript by forming RNA-RNA duplexes with the sense transcript ${ }^{16,17}$. Here we showed that EVA1A-AS altered the recruitment of U2 snRNP to EVA1A pre-mRNA. Notably, EVA1A-AS nascent RNA contains $4 \mathrm{x}$ UCAUAU which are complementary to the branch point site sequence (AUAUGAU) ${ }^{11}$ recognized by U2 snRNP in the intron 2 of EVA1A. This implies that EVA1A-AS forms double-stranded RNA with EVA1A at the branch point site sequence and thus impair the recruitment of U2 snRNP.

EVA1A is a lysosome and endoplasmic reticulum-associated protein. Overexpression of EVA1A in non-small cell lung cancer cells induces cell cycle arrest at the G2/M phase ${ }^{7}$. EVA1A has also been shown to induce autophagosome formation ${ }^{18}$, and in glioblastoma, EVA1A inhibits cell proliferation by inducing autophagy and apoptosis ${ }^{19}$. Here it has been suggested that miR-125b plays a role in the resistance of HCC cells to chemotherapy via a mechanism involving the downregulation of EVA1A-mediated autophagy ${ }^{20}$. Autophagosome marker, $\mathrm{LC}^{21}$, and EVA1A are only partially co-localized in HepG2 cells (SI Fig. 1B), and the molecular role of EVA1A in autophagosome formation and anti-proliferation in HCC is presently not clear. We show here, that overexpression 


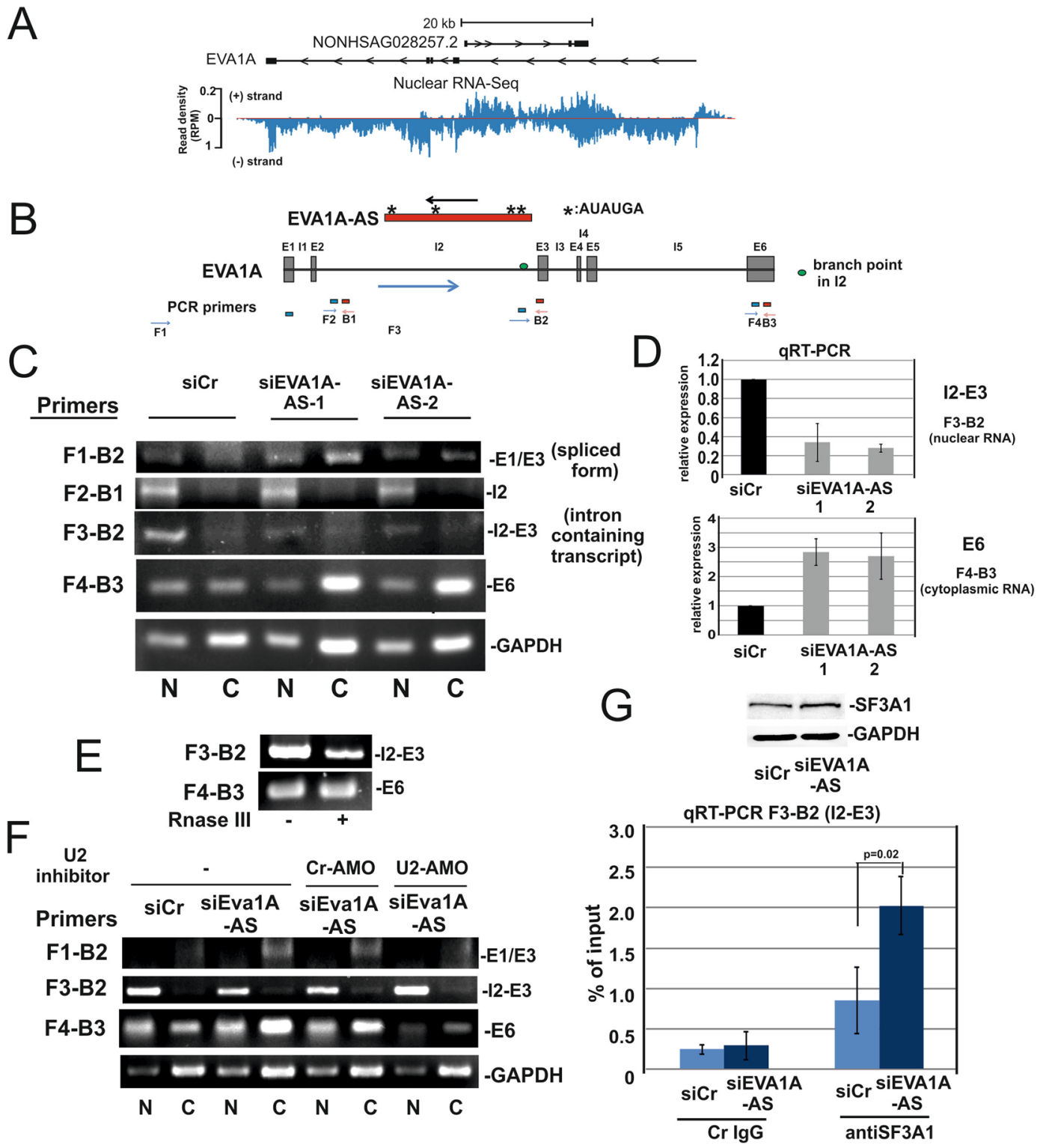

Figure 6. EVA1A-AS suppressed EVA1A expression by inhibiting the splicing of intron 2 of EVA1A. (A) Nuclear RNA-Seq data (ENCFF760IDU) from HepG2 generated by the ENCODE Consortium were aligned to the reference human genome (GRCh38). SeqMonk was used to quantitate and visualize the data. (+) strand: EVA1A-AS; (-) strand: EVA1A. (B) Scheme of EVA1A and EVA1A-AS. Positions of PCR primers are indicated (blue forward; red backward). (C) HepG2 cells were transfected with siCr, siEVA1A-AS-1 or siEVA1A-AS-2. RNAs were isolated from nuclear [N] or cytoplasmic [C] fractions and supplied for RT-PCR or qRT-PCR (D) as indicated. (E) Nuclear RNA was isolated from HepG2 cells, incubated with shortcut RNase III, and RT-PCR was performed using F3-B2 and F4-B3 primers. (F) Sister cultures from (C) were treated with Cr-AMO or U2AMO and RT-PCR was then performed as described in (C). (G) HepG2 cells were transfected with siCr and siEVA1A-AS. Cell extracts were supplied for SF3A1 specific immunoblot or were incubated with control IgG or anti SF3A1 antibody and then precipitated with Protein G Sepharose. Bound RNAs were analyzed by EVA1A, I2-E3 specific qRT-PCR.

of EVA1A causes microtubule catastrophe in G2/M phase. The microtubules play a role in autophagy formation, suggesting that microtubules formation may be influenced by EVA1A.

Notably, the depletion of EVA1A-AS caused the upregulation of endogenous EVA1A and concomitant accumulation of lipid droplets in the cytoplasm. Endogenous EVA1A localized with a similar distribution to that of lipid droplets. It has been shown that in the budding yeast system lipid droplets maintain lipid homeostasis during anaphase for efficient cell separation ${ }^{22}$. Upon treatment with CDK1 inhibitor RO3306, lipid droplets accumulated in the cytoplasm within $24 \mathrm{~h}$, suggesting that lipid droplet accumulation may be an indirect effect of EVA1A. The exact relationship between EVA1A, lipid droplets, and HCC mitosis still remains to be studied. 
Although 161 putative driver genes are associated with 11 recurrently altered pathways in HCC develop$\mathrm{ment}^{23}$, these mutations were not observed in chronic hepatitis or cirrhosis (preneoplastic stages). Interestingly, $28 \%$ of the altered gene products play a role in chromatin remodeling, suggesting that a subset of genes expresses only in HCC but not in normal hepatocytes ${ }^{23}$. Conversely, genes that are expressed in normal liver were not expressed in HCC. Here, products from coding RNAs that are targets of long antisense noncoding RNA may be suitable negative biomarkers for early stages of HCC. Since many long noncoding RNAs are expressed in cell type specific manner, long antisense noncoding RNAs in cancer cells may be promising targets for therapy as well as useful tools to detect novel cancer type specific negative biomarkers.

\section{Methods}

Cell culture, siRNA, and transfection. HepG2 and Huh7 cells were grown in DMEM supplemented with $10 \%$ FCS. RK-13 cells were grown in EMEM supplemented with 10\% FCS.

siMyc (sc-29226) was from Santa Cruz technology (Santa Cruz, CA, USA). Control siRNA-1 (5'-UAAGGCUAUGAA GAGAUAC-3'), siMax (5'-GAAUUGUCUUGCAAGUUAA-3'), siMxil (5'-CACUUGGUUUGCUCAACAA-3'), siEVA1A (5'-CCUUUUUGGCCAAAU GUAU-3'), siEVA1A-AS-1 (5'-UCCUCUAAUUCAGAAAUUUCU-3'), and siEva1-AS-2 (5'-CUCCAGUAUAACAUAAAGUGU-3') were from Microsynth AG (Balgach, Switzerland). GFP-EVA1A and Myc tagged EVA1A plasmids were kindly provided by Prof. Chen (Peking University, China) ${ }^{18}$. Plasmid pmRFP-LC ${ }^{24}$ was from Addgene (Cambridge, MA, USA). Lentivirally modified shTHOC5 and shCr (non-targeting control) HepG2 cells were described previously ${ }^{5}$.

RNA sequencing and data analysis. RNA-seq libraries were generated using the NEB Next Ultra Directional RNA Library Prep Kit for Illumina (NEB). Poly-A-tailed mRNA was isolated from total RNA using oligo-dT beads. Purified mRNA was then fragmented with heat in fragmentation buffer. First strand and second strand cDNA syntheses were performed in accordance with the manufacturer's recommendations. Second strand cDNA was then end-repaired, ligated to an NEBNext Adaptor and individually indexed, followed by limited-cycle (10) amplification. Indexed libraries were pooled and sequenced on an Illumina HiSeq 2500. FASTQ files were generated by CASAVA (v1.8.2). Galaxy workflow for RNA-Seq (www.usegalaxy.org) was used for subsequent data analysis. Reads were mapped to the human reference genome (GRCh38) using Bowtie2 (Galaxy Version 2.3.4.1). The gene expression values (Fragments Per Kilobase Million (FPKM)) were calculated by Cuffdiff (Galaxy Version 2.2.1.5) using the human NONCODEv5 transcript reference. The complete RNA-seq data along with processing protocols have been deposited in NCBI's Gene Expression Omnibus and is accessible through GEO series accession number GSE115139.

Chromatin immunoprecipitation (ChIP). ChIP experiments were performed as previously described ${ }^{25}$. Briefly, in a confluent $10-\mathrm{cm}$ dish, HepG2 cells were crosslinked in $1 \%$ formaldehyde for $5 \mathrm{~min}$ at $37^{\circ} \mathrm{C}$ in the presence of $5 \% \mathrm{CO}_{2}$. Cross-links were quenched in $125 \mathrm{mM}$ glycine ${ }^{25}$. Cells were washed $3 \mathrm{x}$ in ice-cold PBS. Nuclei were fractionated, resuspended in sonication buffer $(15 \mathrm{mM}$ Tris- $\mathrm{HCl} \mathrm{pH} 8.0,0.1 \%$ SDS and $2 \mathrm{mM}$ EDTA) and then sheared into 500-bp DNA fragments using Covaris AFA (Adaptive Focused Acoustics, Woburn, MA, USA) technology according to the manufacturer's instructions ${ }^{25}$. Aliquots of extracts were precipitated pre-coated Protein G Agarose-PLUS (Santa Cruz Biotechnology Inc.) with control IgG, $3 \mu \mathrm{g}$ anti Myc (Santa Cruz Biotechnology Inc.), or Max (Proteintech group, IL, USA) antibodies. Following $2 \mathrm{~h}$ at $4{ }^{\circ} \mathrm{C}$, the beads were washed three times in RIPA buffer, and two times in wash buffer $(500-\mathrm{mM} \mathrm{NaCl}, 1 \% \mathrm{NP} 40,100-\mathrm{mM}$ Tris- $\mathrm{HCl}$ at $\mathrm{pH} 8)^{26}$. Cross-links were reversed for $6 \mathrm{~h}$ at $65^{\circ} \mathrm{C}(250 \mathrm{mM}-\mathrm{NaCl})^{25}$. One microliter of RNase A $(100 \mathrm{mg} / \mathrm{ml}) \mathrm{was}$ added to the bead suspension and incubated for $10 \mathrm{~min}$ at RT. Following proteinase $\mathrm{K}$ digestion at $55^{\circ} \mathrm{C}$ for $1 \mathrm{~h}$, the bound DNA fraction was isolated using NucleoSpin Extract II (Macherey-Nagel, Dueren, Germany) ${ }^{25}$. PCR was then performed using EVA1A or EVA1A-AS promoter-specific primers as shown in Table 1 (ChIP).

Reporter assay. Promoter regions of EVA1 (-1-478) and EVA1A-AS (-1-988) were cloned into pGL2-basic vector. pcDNA3.1+/C-(K)-DYK-MYC was purchased from Genscript (NJ,USA). For luciferase assays, RK-13 cells were seeded on a 96-well plate and $0.5 \mu \mathrm{g}$ of plasmids were cotransfected using Lipofectamine 2000 (Thermo Fisher Scientific, MA, USA). The cells were incubated for $36 \mathrm{~h}$ in complete medium. Luciferase assay was performed using Steady-Glo Luciferase Assay System (Promega) according to manufacturer's protocol. $\beta$-galactosidase activities were measured using Beta-Glo Assay System (Promega). The relative luciferase activities were normalized to the $\beta$-galactosidase activities.

Propidium isodide labelling followed by FACS analysis. HepG2 cells were trypsinized and fixed in ice-cold ethanol. Then fixed cells were then stained in propidium iodide (PI) solution $(100 \mu \mathrm{g} / \mathrm{ml} \mathrm{PI,} 100 \mu \mathrm{g} / \mathrm{ml}$ RNAse, $0.05 \%$ Triton X-100) and analysed by Accuri-C6 flow cytometer (BD Biosciences) under standard settings for detecting PI (FL-2) using a $488 \mathrm{~nm}$ laser.

Immunofluorescence and immunohistochemistry. Immunofluorescence was performed as previously described ${ }^{27}$. TUNEL staining using in situ cell death detection kit (Roche Diagnostics, Mannheim, Germany) was performed according to the manufacturer's instructions. Counterstaining was performed using 4',6-diamidin-2-phenylindole (DAPI). Immunohistochemical studies were performed as detailed previously ${ }^{5}$. Rabbit monoclonal anti Ki67 was purchased from Thermo Sientific (MA, USA).

Immunoblotting procedures. Details of immunoblotting have been described previously ${ }^{28}$. Monoclonal antibody against GAPDH was purchased from Santa Cruz Biotechnology (Santa Cruz, USA). Rabbit polyclonal anti EVA1A antibody was obtained from MyBioSource.Inc (San Diego, CA, USA). 
Corresponding proteins were visualized by incubation with peroxidase conjugated anti-rabbit or anti-mouse immunoglobulin (Santa Cruz Biotechnology) followed by incubation with SuperSignal West FemtoMaximum Sensitivity Substrate (Pierce, Rockford, IL, USA). Results were documented on a LAS4000 imaging system (GE Healthcare Bio-Sciences, Uppsala, Sweden $)^{6,29,30}$.

Semi-quantitative RT-PCR and qRT-PCR analysis. Human normal hepatocyte RNA was purchased from Origene (Maryland, USA). RNA was isolated from cells with the High Pure RNA Isolation kit (Roche Diagnostics) according to the manufacturer's instructions ${ }^{25,27,28} .1 \mu \mathrm{g}$ of RNA was reverse-transcribed using oligo dT primer and the Omniscript reverse transcriptase kit (Qiagen, Hilden, Germany) following the instructions provided. One-twentieth of the cDNA mix was used for real-time PCR using 10 pmol of forward and reverse primer and ORA qPCR Green Rox kit (HighQu, Kraichtal, Germany) in a Qiagen Rotorgene machine ${ }^{25}$. The levels of mRNA expression were standardized to the glyceraldehyde- 3 phosphate dehydrogenase (GAPDH) mRNA level ${ }^{25}$. Primer pairs for each PCR are described in supplemental information Table 1.

RNA immunoprecipitation (RIP) assay. SF3A1- EVA1A-AS complex: HepG2 cells were lysed with lysis buffer ( $10 \mathrm{mM}$ Tris, $150 \mathrm{mM} \mathrm{NaCl}, 1 \mathrm{mM}$ PMSF, $0.5 \%$ NP40, protease inhibitor cocktail (Sigma-Aldrich) and RNase inhibitor ${ }^{31}$. After centrifugation, supernatants were incubated with control IgG or anti SF3A1 antibody (Bethyl, TX, USA), and then precipitated with Protein G Sepharose. Bound RNAs were analyzed by RT-PCR ${ }^{27}$.

Double-stranded RNA assay. The nuclear fraction from HepG2 cells was suspended in $200 \mu \mathrm{l}$ of RIPA buffer $\left(150 \mathrm{mM} \mathrm{NaCl}, 1 \% \mathrm{NP} 40,0.1 \%\right.$ SDS, $20 \mathrm{mM} \mathrm{MnCl}_{2}, 50 \mathrm{mM}$ Tris-Cl at pH 8, $5 \mathrm{mM}$ EDTA at pH 8$)$ and then frozen and thawed three times. After centrifugation, 10 units of Shortcut RNAse III (NEB) which specifically digests double-stranded RNA were added and incubated at $37^{\circ} \mathrm{C}$ for 30 minutes. RNAs were then isolated using the ReliaPrep miRNA Cell and Tissue Miniprep System according to the manufacturer's protocol. cDNA synthesis was carried out using ProtoScript II First Strand cDNA Synthesis Kit (NEB) and Oligo d(T)/ random primers mix (NEB).

TCGA data. Liver Hepatocellular Carcinoma (TCGA, Provisional cohort) was used for the study. Gene expression quantification data of primary HCCs were downloaded from "GDC Data Portal" (https://portal.gdc. cancer.gov/). Mutation was analyzed using an online tool of the GDC portal.

Statistical analysis and limitation of the study. Cell experiments were performed in triplicate and a minimum of three independent experiments were evaluated ${ }^{6,25,32}$. Data were reported as the mean value $+/-$ standard deviation $(\mathrm{SD})^{6,27}$. The statistical significance of the difference between groups was determined by the Student's test (two-sided) ${ }^{6}$. Primary 366 HCC data gathering was limited by the availability from the cancer genome atlas (TCGA) data (https://cancergenome.nih.gov/) 6 .

\section{Data availability}

All data generated or analyzed during this study are included in this published article and its additional files.

Received: 22 July 2019; Accepted: 22 October 2019;

Published online: 21 November 2019

\section{References}

1. Iyer, M. K. et al. The landscape of long noncoding RNAs in the human transcriptome. Nature genetics 47, 199-208, https://doi. org/10.1038/ng.3192 (2015).

2. Yang, Y. et al. Recurrently deregulated lncRNAs in hepatocellular carcinoma. Nature communications 8, 14421, https://doi. org/10.1038/ncomms14421 (2017).

3. Consortium, E. P. et al. Identification and analysis of functional elements in $1 \%$ of the human genome by the ENCODE pilot project. Nature 447, 799-816, https://doi.org/10.1038/nature05874 (2007).

4. Evans, J. R., Feng, F. Y. \& Chinnaiyan, A. M. The bright side of dark matter: IncRNAs in cancer. The Journal of clinical investigation 126, 2775-2782, https://doi.org/10.1172/JCI84421 (2016).

5. Saran, S. et al. Depletion of three combined THOC5 mRNA export protein target genes synergistically induces human hepatocellular carcinoma cell death. Oncogene, https://doi.org/10.1038/onc.2015.433 (2015).

6. Tran, D. D. H. et al. Myc target gene, long intergenic noncoding RNA, Linc00176 in hepatocellular carcinoma regulates cell cycle and cell survival by titrating tumor suppressor microRNAs. Oncogene 37, 75-85, https://doi.org/10.1038/onc.2017.312 (2018).

7. Xie, H. et al. Adenovirus vector-mediated FAM176A overexpression induces cell death in human H1299 non-small cell lung cancer cells. BMB reports $47,104-109$ (2014).

8. Gardner, M. K., Zanic, M. \& Howard, J. Microtubule catastrophe and rescue. Current opinion in cell biology 25, 14-22, https://doi. org/10.1016/j.ceb.2012.09.006 (2013).

9. Zervos, A. S., Gyuris, J. \& Brent, R. Mxi1, a protein that specifically interacts with Max to bind Myc-Max recognition sites. Cell 72, 223-232 (1993)

10. Jiang, K., Hein, N., Eckert, K., Luscher-Firzlaff, J. \& Luscher, B. Regulation of the MAD1 promoter by G-CSF. Nucleic acids research 36, 1517-1531, https://doi.org/10.1093/nar/gkn002 (2008)

11. Zhang, Q. et al. BPP: a sequence-based algorithm for branch point prediction. Bioinformatics 33, 3166-3172, https://doi. org/10.1093/bioinformatics/btx401 (2017).

12. Sanchez-Vega, F. et al. Oncogenic Signaling Pathways in The Cancer Genome Atlas. Cell 173, 321-337 e310, https://doi. org/10.1016/j.cell.2018.03.035 (2018).

13. Zhang, T. et al. Overexpression of flavin-containing monooxygenase 5 predicts poor prognosis in patients with colorectal cancer. Oncology letters 15, 3923-3927, https://doi.org/10.3892/ol.2018.7724 (2018).

14. Scherneck, S. et al. Positional cloning of zinc finger domain transcription factor Zfp69, a candidate gene for obesity-associated diabetes contributed by mouse locus Nidd/SJL. PLoS genetics 5, e1000541, https://doi.org/10.1371/journal.pgen.1000541 (2009).

15. Srivastava, K., Lee, E., Owens, E., Rujirojindakul, P. \& Flegel, W. A. Full-length nucleotide sequence of ERMAP alleles encoding Scianna (SC) antigens. Transfusion 56, 3047-3054, https://doi.org/10.1111/trf.13801 (2016). 
16. Villamizar, O., Chambers, C. B., Riberdy, J. M., Persons, D. A. \& Wilber, A. Long noncoding RNA Saf and splicing factor 45 increase soluble Fas and resistance to apoptosis. Oncotarget 7, 13810-13826, https://doi.org/10.18632/oncotarget.7329 (2016).

17. Beltran, M. et al. A natural antisense transcript regulates Zeb2/Sip1 gene expression during Snaill-induced epithelial-mesenchymal transition. Genes \&. development 22, 756-769, https://doi.org/10.1101/gad.455708 (2008).

18. Hu, J. et al. TMEM166/EVA1A interacts with ATG16L1 and induces autophagosome formation and cell death. Cell death \& disease 7, e2323, https://doi.org/10.1038/cddis.2016.230 (2016).

19. Shen, X. et al. EVA1A inhibits GBM cell proliferation by inducing autophagy and apoptosis. Experimental cell research 352, 130-138, https://doi.org/10.1016/j.yexcr.2017.02.003 (2017).

20. Ren, W. W. et al. MicroRNA-125b reverses oxaliplatin resistance in hepatocellular carcinoma by negatively regulating EVA1A mediated autophagy. Cell death \& disease 9, 547, https://doi.org/10.1038/s41419-018-0592-z (2018).

21. Munafo, D. B. \& Colombo, M. I. A novel assay to study autophagy: regulation of autophagosome vacuole size by amino acid deprivation. Journal of cell science 114, 3619-3629 (2001).

22. Yang, P. L., Hsu, T. H., Wang, C. W. \& Chen, R. H. Lipid droplets maintain lipid homeostasis during anaphase for efficient cell separation in budding yeast. Molecular biology of the cell 27, 2368-2380, https://doi.org/10.1091/mbc.E16-02-0106 (2016).

23. Schulze, K. et al. Exome sequencing of hepatocellular carcinomas identifies new mutational signatures and potential therapeutic targets. Nature genetics 47, 505-511, https://doi.org/10.1038/ng.3252 (2015).

24. Kimura, S., Noda, T. \& Yoshimori, T. Dissection of the autophagosome maturation process by a novel reporter protein, tandem fluorescent-tagged LC3. Autophagy 3, 452-460 (2007).

25. Tran, D. D. et al. THOC5 controls $3^{\prime}$ end-processing of immediate early genes via interaction with polyadenylation specific factor 100 (CPSF100). Nucleic acids research 42, 12249-12260, https://doi.org/10.1093/nar/gku911 (2014).

26. Davidson, L., Muniz, L. \& West, S. $3^{\prime}$ end formation of pre-mRNA and phosphorylation of Ser2 on the RNA polymerase II CTD are reciprocally coupled in human cells. Genes \& development 28, 342-356, https://doi.org/10.1101/gad.231274.113 (2014).

27. Tran, D. D. et al. Transcriptional regulation of immediate-early gene response by THOC5, a member of mRNA export complex, contributes to the M-CSF-induced macrophage differentiation. Cell death \& disease 4, e879, https://doi.org/10.1038/cddis.2013.409 (2013).

28. Tran, D. D. et al. Treatment with MAPKAP2 (MK2) inhibitor and DNA methylation inhibitor, 5-aza dC, synergistically triggers apoptosis in hepatocellular carcinoma (HCC) via tristetraprolin (TTP). Cellular signalling 28, 1872-1880, https://doi.org/10.1016/j. cellsig.2016.09.002 (2016).

29. Guria, A. et al. Identification of mRNAs that are spliced but not exported to the cytoplasm in the absence of THOC5 in mouse embryo fibroblasts. Rna 17, 1048-1056, https://doi.org/10.1261/rna.2607011 (2011).

30. Tran, D. D., Koch, A. \& Tamura, T. THOC5, a member of the mRNA export complex: a novel link between mRNA export machinery and signal transduction pathways in cell proliferation and differentiation. Cell communication and signaling: CCS 12, 3, https://doi. org/10.1186/1478-811X-12-3 (2014).

31. Ramachandran, S. et al. An ataxia-telangiectasia-mutated (ATM) kinase mediated response to DNA damage down-regulates the mRNA-binding potential of THOC5. Rna 17, 1957-1966, https://doi.org/10.1261/rna.2820911 (2011).

32. Tamura, T. et al. FMIP, a novel Fms-interacting protein, affects granulocyte/macrophage differentiation. Oncogene 18, 6488-6495, https://doi.org/10.1038/sj.onc.1203062 (1999).

\section{Acknowledgements}

We thank C. Bruce Boschek for critically reading the manuscript. This work is a part of thesis of SEN and AA. This research was supported by DFG Ta-111/13-3 to T.T., Niedersächsische Krebsgesellschaft to DDHT, PhD program Molecular Medicine in HBRS and Leistungsorientierte Mittelvergabe with Frauenfaktor from $\mathrm{MHH}$. We acknowledge support by the German Research Foundation (DFG) and the Open Access Publication Fund of Hannover Medical School (MHH).

\section{Author contributions}

D.D.H.T. and T.T. conceived and designed the experiments. S.E.N., A.A. and D.D.H.T. performed the experiments, S.E.N., D.D.H.T., A.H., L.W. analyzed the data. D.D.H.T. and T.T. wrote the manuscript. All authors have read and approved the manuscript.

\section{Competing interests}

The authors declare no competing interests.

\section{Additional information}

Supplementary information is available for this paper at https://doi.org/10.1038/s41598-019-53944-2.

Correspondence and requests for materials should be addressed to D.D.H.T.

Reprints and permissions information is available at www.nature.com/reprints.

Publisher's note Springer Nature remains neutral with regard to jurisdictional claims in published maps and institutional affiliations.

(c) (i) Open Access This article is licensed under a Creative Commons Attribution 4.0 International

License, which permits use, sharing, adaptation, distribution and reproduction in any medium or format, as long as you give appropriate credit to the original author(s) and the source, provide a link to the Creative Commons license, and indicate if changes were made. The images or other third party material in this article are included in the article's Creative Commons license, unless indicated otherwise in a credit line to the material. If material is not included in the article's Creative Commons license and your intended use is not permitted by statutory regulation or exceeds the permitted use, you will need to obtain permission directly from the copyright holder. To view a copy of this license, visit http://creativecommons.org/licenses/by/4.0/.

(c) The Author(s) 2019 